\title{
A novel porcine acellular dermal matrix scaffold used in periodontal regeneration
}

\author{
Jing Guo ${ }^{1,2}$, Hui Chen ${ }^{1,2}$, Ying Wang ${ }^{3}$, Cheng-Bo Cao ${ }^{4}$ and Guo-Qiang Guan ${ }^{3}$
}

Regeneration of periodontal tissue is the most promising method for restoring periodontal structures. To find a suitable bioactive threedimensional scaffold promoting cell proliferation and differentiation is critical in periodontal tissue engineering. The objective of this study was to evaluate the biocompatibility of a novel porcine acellular dermal matrix as periodontal tissue scaffolds both in vitro and in vivo. The scaffolds in this study were purified porcine acellular dermal matrix (PADM) and hydroxyapatite-treated PADM (HA-PADM). The biodegradation patterns of the scaffolds were evaluated in vitro. The biocompatibility of the scaffolds in vivo was assessed by implanting them into the sacrospinal muscle of $\mathbf{2 0}$ New Zealand white rabbits. The hPDL cells were cultured with PADM or HA-PADM scaffolds for 3, 7, 14, 21 and 28 days. Cell viability assay, scanning electron microscopy (SEM), hematoxylin and eosin (H\&E) staining, immunohistochemistry and confocal microscopy were used to evaluate the biocompatibility of the scaffolds. In vitro, both PADM and HA-PADM scaffolds displayed appropriate biodegradation pattern, and also, demonstrated favorable tissue compatibility without tissue necrosis, fibrosis and other abnormal response. The absorbance readings of the WST-1 assay were increased with the time course, suggesting the cell proliferation in the scaffolds. The hPDL cells attaching, spreading and morphology on the surface of the scaffold were visualized by SEM, H\&E staining, immnuohistochemistry and confocal microscopy, demonstrated that hPDL cells were able to grow into the HA-PADM scaffolds and the amount of cells were growing up in the course of time. This study proved that HA-PADM scaffold had good biocompatibility in animals in vivo and appropriate biodegrading characteristics in vitro. The hPDL cells were able to proliferate and migrate into the scaffold. These observations may suggest that HA-PADM scaffold is a potential cell carrier for periodontal tissue regeneration.

International Journal of Oral Science (2013) 5, 37-43; doi:10.1038/ijos.2013.1; published online 15 March 2013

Keywords: periodontal ligament cells; periodontal regeneration; porcine acellular dermal matrix; scaffold; tissue engineering

\section{INTRODUCTION}

Periodontitis is an inflammatory disease invading the periodontium including gingival, periodontal ligament, cementum and alveolar bone, which could affect approximately $50 \%$ of adults, with as much as $10 \%-30 \%$ patients displaying bone resorption and ultimately tooth loss. ${ }^{1}$ The current periodontal treatments are generally succeed in removing the pathogenic bacterial and arrest the progress of periodontal disease destruction. ${ }^{2}$ This includes the formation of a new connective tissue attachment, new cementum and supporting bone. ${ }^{3}$ However, to restore the structure and function of the periodontium is still a challenge for dental professionals. With the recent advance in stem cell research and bioengineering technology, periodontal tissue regeneration became the most promising method for restoring periodontal structures. ${ }^{4}$ In periodontal tissue regeneration, a biodegradable scaffold for the delivery of cells to the wound site, as well as to preserve the space for the new periodontal tissue formation, is necessary. ${ }^{5-6}$ The ideal scaffold for periodontal tissue engineering should be relatively easy to handle, allowing the incorporation of cells, allowing the free diffusion of cells and growth factors, permitting the establishment of a vascular bed to ensure survival of the implanted cells, inducing a minimal inflammatory response and biodegraded. ${ }^{7}$ To date, no designed scaffolds meet all these criteria. ${ }^{8}$

The concerns of the current available scaffolds include unforeseeable cell-biomaterial interactions, uneven degradation of the biomaterial, inflammatory reaction and limited cell seeding efficiency. In this research, we focus on a native three-dimenssional collagen framework, purified from porcine acellular dermal matrix (PADM), and further treated with hydoroxyapatite (HA) to promote bioactivity and cell differentiation. The hydroxyapatite-porcine acellular dermal matrix (HA-PADM) scaffold has two-level pore structure, with large channels (approximately $100 \mu \mathrm{m}$ in diameter) inherited from the purified PADM microstructure and small pores (less than $100 \mathrm{~nm}$ in diameter) formed by self-assembled HA on the channel surfaces. The objective of this study was to evaluate the biocompatibility of a novel porcine acellular dermal matrix as periodontal tissue scaffolds both in vitro and in vivo.

\section{MATERIALS AND METHODS}

Preparation of porcine skin scaffolds

Fresh porcine skin was purchased from a local slaughterhouse. The preparation method of the PADM scaffold is described in our patent 
(CN03139063.3). Briefly, after complete cleaning, excision of the subdermal fat tissue, and removal of hair, the skin was cut into pieces with a thickness of $1.0 \mathrm{~mm}$ and purified through basic processing and enzymatic extraction methods to remove the fat and cells. The product was washed carefully with distilled water, and the moisture laden purified porcine skin was frozen at $-80{ }^{\circ} \mathrm{C}$. After lyophilization at $-60{ }^{\circ} \mathrm{C}$ for $4 \mathrm{~h}$, a PADM framework was obtained.

\section{Preparation of hydroxyapatite treated PADM}

First, PADM framework was cut into $10 \mathrm{~mm} \times 10 \mathrm{~mm} \times 1 \mathrm{~mm}$ flakes. Then 20 pieces of the above PADM were precalcified through rinsing in $400 \mathrm{~mL}$ of $0.1 \mathrm{~mol} \cdot \mathrm{L}^{-1} \mathrm{CaCl}_{2}, \mathrm{~K}_{2} \mathrm{HPO}_{4}$ and $\mathrm{CaCl}_{2}$ solution for $24 \mathrm{~h}$ in an incubation shaker $\left(120 \mathrm{~g}, 37^{\circ} \mathrm{C}\right)$ by using an alternative soaking method. Washing with double deionized $\mathrm{H}_{2} \mathrm{O}$ for 20 min was needed between every two steps to remove the free and weakly connected $\mathrm{Ca}^{2+}$ and $\mathrm{HPO}_{4}{ }^{2-}$. Finally, the precalcification PADM framework was mineralized in $1 \mathrm{~L}$ simulated body fluid (SBF) in an incubation box at $37^{\circ} \mathrm{C}$ for $4,8,15$ and 30 days to obtain the HA-PADM scaffolds. The HA-PADM scaffolds were washed completely and lyophilized at $-60{ }^{\circ} \mathrm{C}$ for $4 \mathrm{~h}$ in a vacuum lyophilizer.

\section{Sterilization of the materials}

The HA-PADM and PADM were cut into $5 \mathrm{~mm} \times 5 \mathrm{~mm} \times 1 \mathrm{~mm}$ square flakes and sterilized with ethylene oxide (EO) gas for $30 \mathrm{~min}$.

\section{Degradable test on the novel developed scaffolds}

Three kinds of the same size (weight: approximate $5 \mathrm{mg}$ ) EO sterilized materials (PADM group, HA-PADM group and gelfoam group) were put in cell culture medium (DMEM) in an incubation box of a $37{ }^{\circ} \mathrm{C}$, $5 \% \mathrm{CO}_{2}$ humidified atmosphere. The materials were given fresh media every 3 days. After 1, 2, 3 and 4 weeks, they were studied on their weight, degrading rate. Gelfoam (Ferrosan A/S, Soeborg, Denmark) was used as a control group. For each group, there were 20 specimens and the experiment was repeated three times.

\section{Biocompatibility of the novel developed scaffolds}

Twenty adult New Zealand white rabbits (no limit of male or female) with an average weight of $1-1.5 \mathrm{~kg}$, aged from 3 to 4 months were used for the experiment. A longitudinal skin incision was made in the sacrospinal muscle symmetrically under general anesthesia. Three kinds of the same size $(10 \mathrm{~mm} \times 3 \mathrm{~mm} \times 1 \mathrm{~mm})$ EO sterilized scaffolds (PADM group, HA-PADM group and gelfoam Group) were implanted into the dorsal muscles of rabbits in vivo. Four rabbits were killed every time at 3, 7, 14, 21 and 28 days. Retrieved grafts were fixed in $10 \%$ buffered formalin, embedded in paraffin, and stained with hematoxylin and eosin ( $\mathrm{H} \& \mathrm{E})$. The specimens were analyzed for the degree of inflammation and fibrosis. Gelfoam was used as a control group. The animal research protocol was approved by the Animal Care and Use Committee of Shandong University.

\section{Human periodontal ligament cells cultures}

The human periodontal ligament (hPDL) cells were isolated from human extracted teeth described previously. ${ }^{9}$ The cells were maintained in minimal essential medium with $10 \%$ fetal bovine serum and antibiotic, and kept in a humidified incubator at $37{ }^{\circ} \mathrm{C}$ in $5 \% \mathrm{CO}_{2}$ atmosphere.

\section{Encapsulation of hPDL cells on the procine dermal scaffolds}

The hPDL Cells were used at passages 2 to 4 in experiments. After $90 \%$ confluence, cells were digested by $0.25 \%$ trypsin and cell density was adjusted to $1 \times 10^{5}$ cells $\cdot \mathrm{mL}^{-1}$. The $5 \mathrm{~mm} \times 5 \mathrm{~mm} \times 1 \mathrm{~mm}$ scaffolds flakes were first placed into 48 -well plastic culture plates, one piece per well. Then $0.2 \mathrm{~mL}$ hPDL cells suspension were dropped onto the dried scaffolds. After the scaffolds were soaked, another $0.3 \mathrm{~mL} \alpha$-minimal essential medium medium ( $10 \%$ fetal bovine serum) was added to make up a total of $0.5 \mathrm{~mL}$ medium for each well. The cells were kept in a humidified incubator at $37{ }^{\circ} \mathrm{C}$ in $5 \% \mathrm{CO}_{2}$ atmosphere. The medium were changed every 2-3 days. Gelfoam was again used as a control scaffold.

\section{Cell viability and cytotoxicity assays}

In order to evaluate the scaffolds cytotoxicity, the hPDL cells were randomly divided into blank, gelfoam, HA-PADM, PADM groups $(n=9)$. The hPDL cells were cultured with scaffolds for 1,3 and 6 days. Cell proliferation reagent water-soluble tetrazolium-1 (WST1) (Roche Applied Science, Indianapolis, IN, USA) was used to detect the metabolic activity of the cells. At each time point, WST-1 was added to the culture media present in the wells. Cells were incubated with the reagent for $3 \mathrm{~h}$ in a $37{ }^{\circ} \mathrm{C}, 5 \% \mathrm{CO}_{2}$ environment, after which, $100 \mathrm{~mL}$ developed media/reagent from each well was transferred to new 96-well plates and absorbance was read by using a microplate reader AD340 (Beckman coulter Inc., Brea, CA, USA) at $450 \mathrm{~nm}$. This experiment was carried out three times.

\section{Scanning electron microscopy}

The method of scanning electron microscopy (SEM) was used to examine the morphological characteristics of hPDL cells cultured onto the scaffold. The hPDL cells cultured on the scaffolds for 1, 3 and 6 days at $37{ }^{\circ} \mathrm{C}$ in a $\mathrm{CO}_{2}$ incubator. At each time point, the scaffolds with cells washed twice with phosphate-buffered saline (PBS). Then the cells were fixed with $2 \%$ glutaraldehyde in $0.1 \mathrm{~mol} \cdot \mathrm{L}^{-1}$ sodium cacodylate buffer for $1 \mathrm{~h}$ at room temperature. After being rinsed with $0.1 \mathrm{~mol} \cdot \mathrm{L}^{-1}$ sodium cacodylate buffer for $15 \mathrm{~min} 3$ times, scaffolds were dehydrated with graded series of ethanol solutions (35\%, $50 \%$, $70 \%, 80 \%, 95 \%$ and $100 \%$ each for $20 \mathrm{~min}$ ) and at the end add hexamethyldisazane for $10 \mathrm{~min}$. The samples were air dried and mounted on stubs, sputtered carbon coat and examined by SEM (Hitachi SU-70; Hitachi Instruments, Schaumburg, IL, USA).

\section{Histology and immunohistochemsitry}

For histological study, the cells cultured on the scaffolds were fixed with $4 \%$ formaldehyde in PBS for $24 \mathrm{~h}$ before processed to paraffin embedding. The sections were stained with $\mathrm{H} \& \mathrm{E}$, then imagines were taken with light microcopy (Olympus BX51; Olympus Optical Co., Ltd, Tokyo, Japan).

For immunohistochemical study, frozen sections from scaffold or cells cultured on slides, were fixed with $4 \%$ formaldehyde in PBS for 30 min. The sections were blocked with $10 \%$ normal goat serum in $\mathrm{PBS}$ for $2 \mathrm{~h}$ at room temperature. Primary antibodies used for staining included mouse monoclonal antibodies for collagen III (Chemicon, Temecula, CA, USA) and Tublin- $\alpha$ (Invitrogen, Life Technologies, Carlsbad, CA, USA). Secondary antibody was Alexa Fluor 546 antimouse immunoglobulin G (IgG) (Invitrogen, Life Technologies, Carlsbad, CA, USA). Alexa Fluor 488 Phalloidin and DAPI (Invitrogen, Life Technologies, Carlsbad, CA, USA) were used to stained filamentous actin (F-actin) and nuclear respectively. Slides were observed by using a fluorescence microscope (AX10 Imager.M1; Carl Zeiss Microimaging, Thornwood, NY, USA) or a confocal microscope (Zeiss LSM 510 Meta NLO; Carl Zeiss Microimaging, Thornwood, NY, USA). 

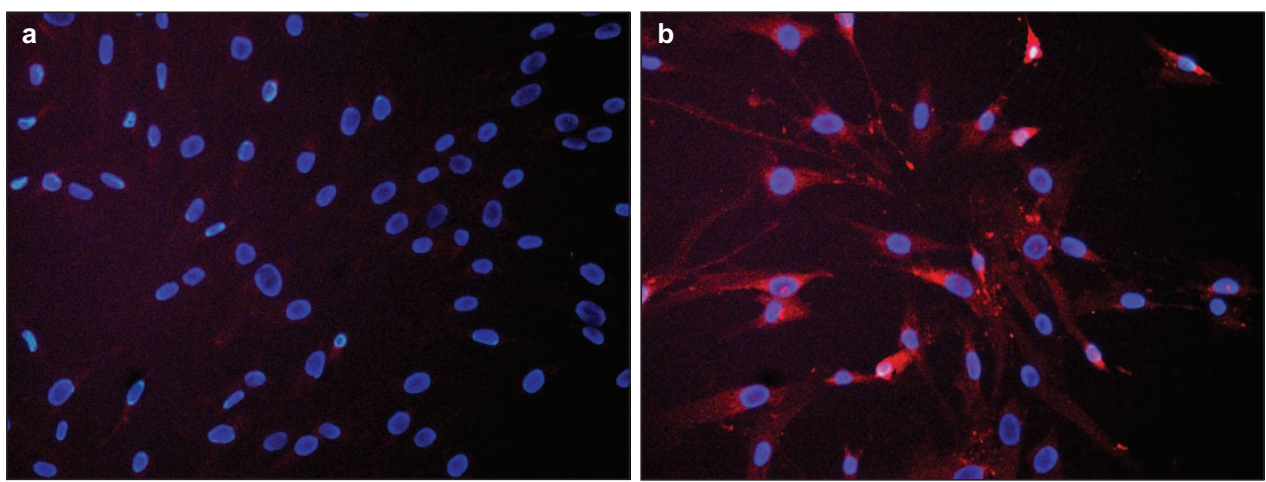

Figure 1 Collagen III staining of hPDL cells. hPDL cells were treated with PBS (a) or anti-Collagen III antibody (b), followed by Alexa Fluor 546 anti-mouse IgG as secondary antibody (red). Cell nuclei were stained with DAPI in light blue. PBS, phosphate-buffered saline; IgG, immunoglobulin G; DAPI, 4',6-diamidino-2-phenylindole.

\section{Statistical analysis}

Data were expressed as the mean \pm s.d. After confirmation of the normal distribution of data by Shapiro-Wilk test, two-way analysis of variance for repeated measurements followed by Tukey's HSD (honestly significant difference) test for multiple comparisons was used in this study. $P \leqslant 0.05$ was considered significantly. All statistical analyses were computed using the Statistical Package for Social Science (SPSS, Inc., Chicago, IL, USA).

\section{RESULTS}

Characterization of hPDL cells

Immunohistochemical staining of hPDL cells on slides indicated that these cells expressed collagen III (Figure 1). Moreover, these cells had a spindle or spindle-like morphology. Collagen III is a periodontial ligament cell marker.

Degradable test on the novel developed scaffold

Overtime, all three materials showed degradation (Figure 2), but among the three groups, biodegradation test showed that HAPADM degraded more slowly than PADM and gelfoam $(P<0.05)$; it did not degraded significantly over 4 -week incubation $(P>0.05)$.

Figure 2 also illustrated that PADM degraded slowly at the first week, then fast in following 2 weeks. The degrading rate of HAPADM was stable; the degrading rate of gelfoam was the fastest, while that of HA-PADM was the slowest among these scaffolds.

\section{Histological analysis on the scaffold in vivo tissue response}

All rabbits survived during the experiment with no complication, such as infection and tissue necrosis in the implanted site (Figure 3). After 3 and 7 days' implantation, histological analysis showed a middle tissue reaction with the infiltration of inflammatory cells consisting of mononuclear leukocytes and macrophages. On the fourteenth day after implantation, the inflammatory cells decreased and angiogenesis was demonstrated by the development of several new vessels inside the matrix. On days 21 and 28, the grafts had a partial degradation and the inflammatory cells nearly disappeared. By contrast, results also showed that the HA-PADM has better biocompatibility. These in vivo tissue compatibility results are also consistent to in vitro cytocompatibility study.

Cytocompatibility analysis on the scaffolds

Figure 4 shows the result of WST-1 assay of the hPDL cells cultured with scaffolds for 1, 3 and 6 days. The optical density (OD) values increased with the time course in cell only, gelfoam and HA-PADM group, but decreased closed to zero in PADM group $(P<0.05)$. gelfoam actually stimulated cell proliferation. However, PADM significantly suppressed cell growth $(P<0.05)$. The OD values of days 3 and 6 were highest in gelfoam group, that was even higher than the values of cell only, suggesting more cells grown with the present of gelfoam $(P<0.05)$. To understanding this finding, we further studied the effect of scaffolds on the morphology of cells by using histological staining, SEM and immunohistochemistry staining.

The demonstration of SEM, confocal microscopy and frozen section immunohistochemistry

In general, cell attachment, spreading and proliferation on the scaffolds reflect the ability of the scaffolds to make contact with the cells.

SEM images (Figure 5) showed hPDL cells attached to the HAPADM scaffold and gelfoam from day 1 . However, the cell morphology on the two scaffolds was totally different. HA-PADM scaffold had a relatively dense microstructure. Cells attached to the HA-PADM scaffold exhibited a star-shaped appearance with multiprocess. The cells were well distributed and well attached on the surface of the HAPADM scaffold. We also observed that the cells communicated with each other by the processes. After incubation for 6 days, cells proliferated a great lot on the surface of HA-PADM scaffold. On the other hand, gelfoam provided a porous three-dimensional network with highly smooth curly sheets. The hPDL cells attached firmly on the sheets in gelfoam, which were always flat and stretched. The connections between cells were not seen as much as those in HA-PADM.

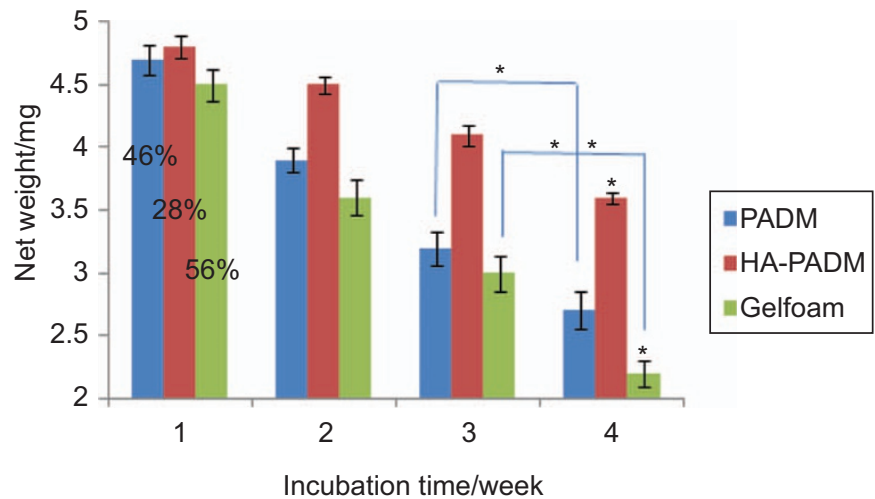

Figure 2 Biodegration rate of three different materials. Degradatopm rates of scaffolds during incubation time are also indicated in figure. $* P<0.05$; $* * P<0.01$. 

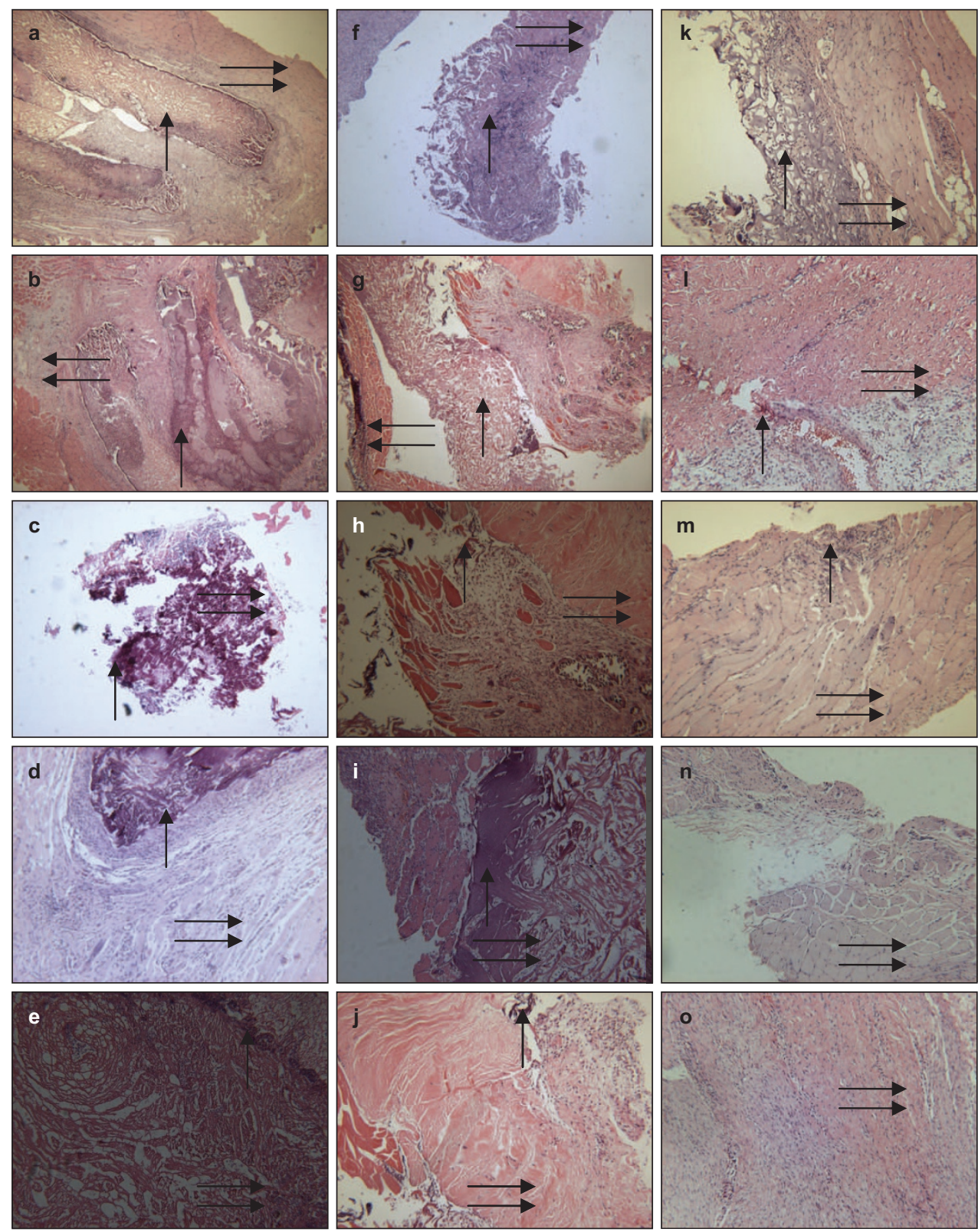

Figure 3 Photomicrograph showing the histological appearance of PADM and HA-PADM in muscle. (a) HA-PADM embedded after 3 days. (b) HA-PADM embedded after 7 days. (c) HA-PADM embedded after 14 days. (d) HA-PADM embedded after 21 days. (e) HA-PADM embedded after 28 days. (f) PADM embedded after 3 days. (g) PADM embedded after 7 days. (h) PADM embedded after 14 days. (i) PADM embedded after 21 days. (j) PADM embedded after 28 days. (k) Gelfoam embedded after 3 days. (I) Gelfoam embedded after 7 days. (m) Gelfoam embedded after 14 days. (n) Gelfoam embedded after 21 days. (o) Gelfaom embedded after 28 days. Single arrow indicates materials; double arrows indicate muscles. HA-PADM, hydroxyapatite-porcine acellular dermal matrix; PADM, porcine acellular dermal matrix.

H\&E staining (Figure 6b), as well as immunohistochemistry staining (Figure 6), provided the cross-section views of the cells cultured with scaffolds. We found that hPDL cells grown on the surface and subsurface of the HA-PADM scaffold (Figure 6a). After prolonged culture, the hPDL cells were able to migrated into the HA-PADM scaffolds at certain spots. At 2 and 4 weeks, we observed that cells proliferated in the center of the HA-PADM scaffolds. The sections of gelfoam showed that hPDL cells lined up on the porous structure. After 4 weeks of culture, the cells filled up the gelfoam scaffold (Figure 6b). These observations were consistent with our WST-1 assay and SEM findings that gelfoam provided much more spaces for cells to grow.
Another interesting finding from the immunohistochemistry staining (Figure $6 \mathrm{~b}$ ) is that the F-action expression level was much higher in the hPDL cells on HA-PADM compare to the cells on gelfoam, indicating that hydoroxyapatite and collagen components of the HA-PADM scaffold promoted hPDL cells differentiation.

\section{DISCUSSION}

Using collagen as a biomaterial for scaffold in tissue engineering has its disadvantages compared to other materials. These include its low biomechanical stiffness and rapid biodegradation. ${ }^{10}$ Further, the high rate of enzymatic degradation of natural collagen in vivo makes stabilization of collagen-based biomaterials necessary. ${ }^{11-12}$ Moreover, for tissue 


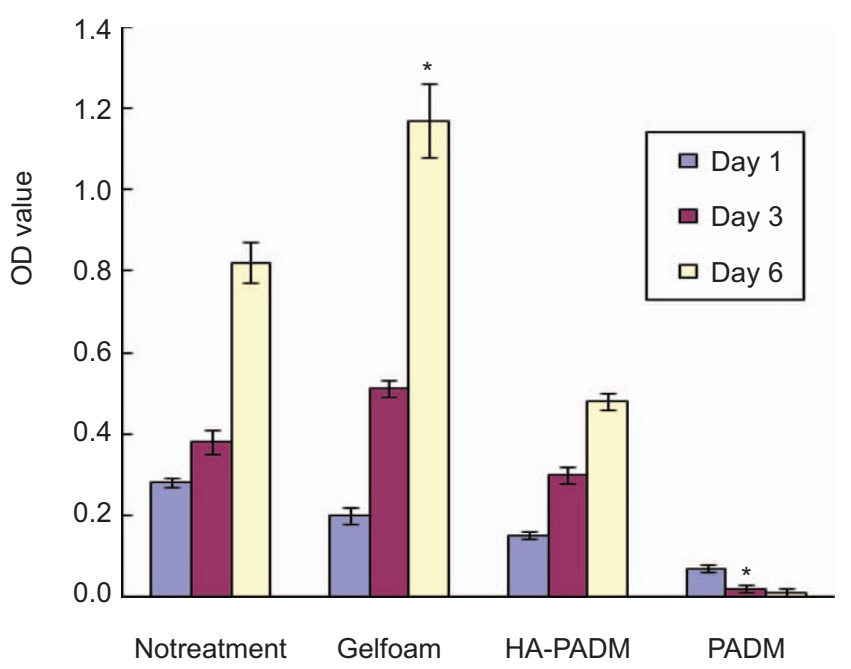

Figure 4 Cytotoxicity of the scaffolds: cellular metabolic activity was measured as a means of detecting cell proliferation at day 1 , day 3 and day 6 . All the cells remained viable and proliferative for control (cell only), Gelfoam and HAPADM group except for pure PADM. The OD value for PADM at day 3 and day 6 were close to zero, indicating almost no live cells were presented in the wells. $n=9$. $* P<0.05$. PADM, porcine acellular dermal matrix; HA-PADM, hydroxyapatite-porcine acellular dermal matrix; $\mathrm{OD}$, optical density.

engineering, previous studies have demonstrated that high mechanical strength is important for biodegradable scaffolds ${ }^{13-15}$ or allogenic acellular matrix scaffolds. ${ }^{16}$ Major disadvantages of biodegradable polymer scaffolds include the requirement of reconstruction of the natural three-dimensional structures, and the absence of important ECM proteins in the synthetic polymers. ${ }^{17}$ Thus, acellularized xenogenic tissues that maintain natural three-dimensional structures are a promising alternative to biodegradable polymer scaffolds. ${ }^{18-19}$

In periodontal therapy, a biodegradable scaffold for delivery of cells to the wound site, as well as for preservation the space of the defect in the new periodontal tissue formation, is required. The optimal biomechanical properties and regenerative capacity remains a challenge for periodontal tissue regeneration. In this study, we evaluated the biocompatibility of an HA-PADM. By using hPDL cells, we test the suitability for the scaffolds in periodontal tissue regeneration. The PADM scaffold exhibited visible toxicity instead of being helpful for cell growth and migration to hPDL cells. The most possible reasons of the cytotoxicity could be the present of residual detergent and or digestive enzyme, which have negative effects on the hPDL cell survival. On the other hand, the HA-PADM scaffold prepared by a two-stop biomimetic mineralization method maintained the natural three-dimensional microstructure, and also eliminated the toxic reagents within the PADM. Another advantage of the hydroxyapatite coating is reducing the degradation rate and improving the mechanical property of the collagen scaffold. Moreover, the HA-PADM showed hydrophilic when we dropping the cell solution on the dried materials. All these properties make the HA-PADM be a promising material for periodontal tissue regeneration.

From the cell proliferation assay, we found more cells grown with Gelfoam, a commercially available gelatin sponge. SEM imaging showed Gelfoam had loose porous structure compare to HA-PADM scaffold, so that it provided more space for cells migration and proliferation. However, on the smooth microsheet structures of Gelfoam, hPDL cells were flat and firmly attached to
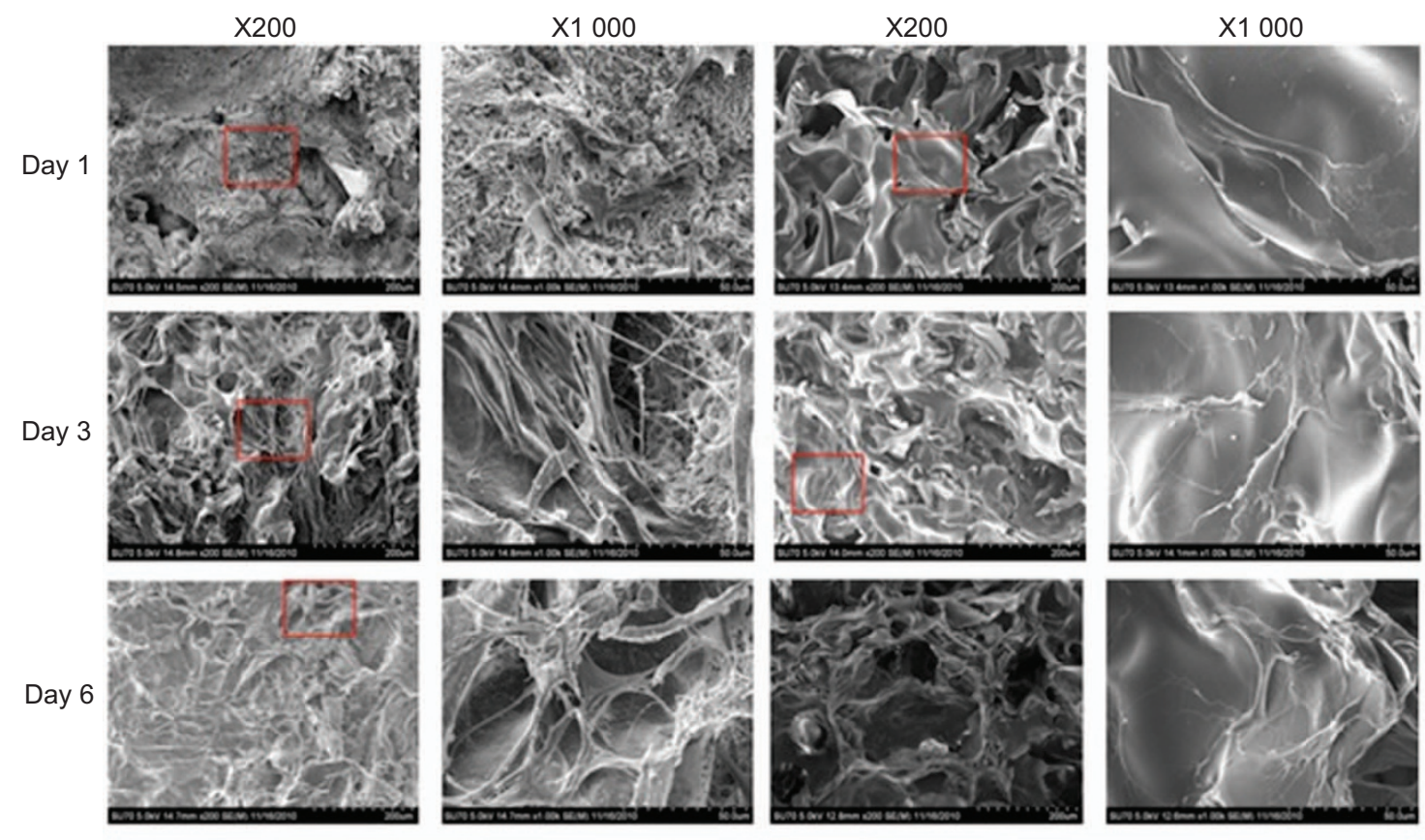

HA-PADM

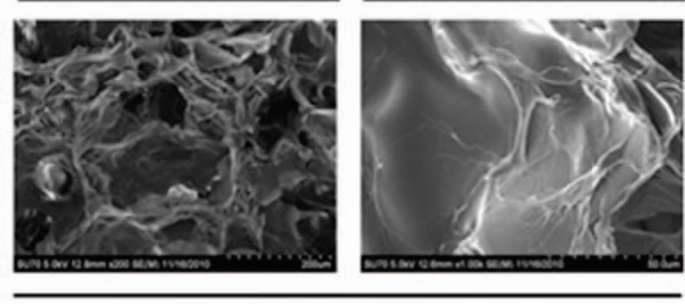

Gelfoam

Figure 5 SEM images showed the morphology of hPDL cells on HA-PADM and gelfoam at day1, day 3 and day 6 . HA-PADM, hydroxyapatite-porcine acellular dermal matrix; SEM, scanning electron microscopy. 
a
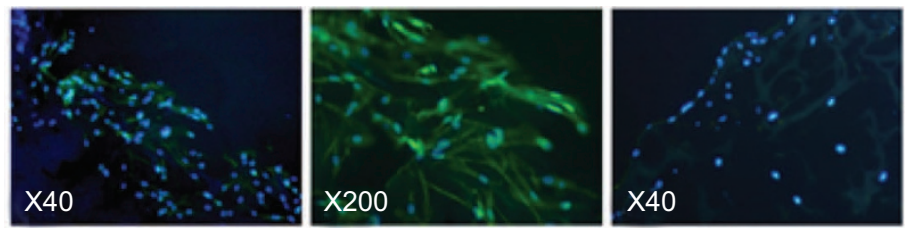

Days 3
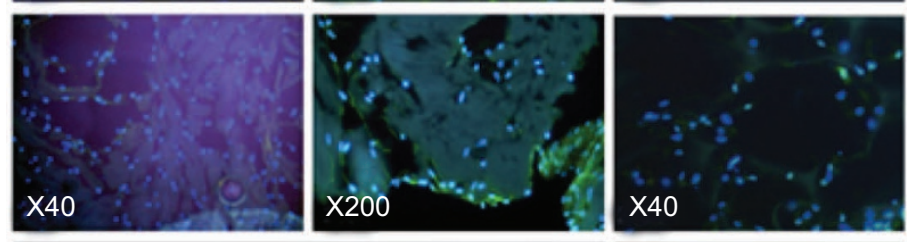

HA-PADM

Gelfoam
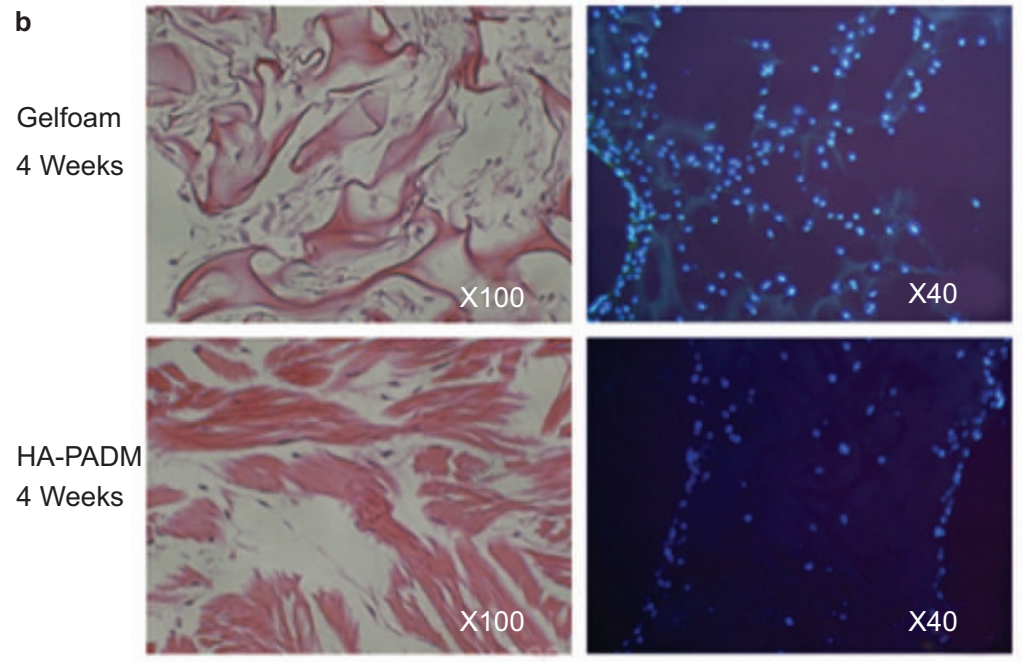

c

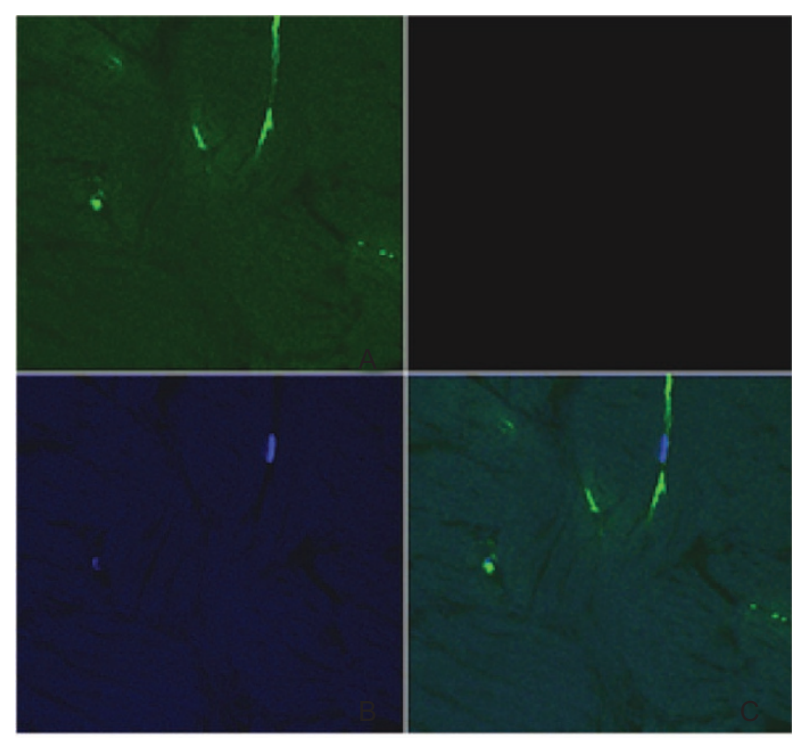

Figure 6 The hPDL proliferation and migration in HA-PADM and gelform. (a) The F-actin fibers were stained in green and nuclei in light blue. At day 3, hPDL cells form multiple layers on the surface of HA-PADM. After 14 days, more cells were present in the subsurface area suggested cells could migrate into the scaffold. Strong Factin expression detected for the cells cultured with HA-PADM, well it is hardly present for the cells cultured with Gelform. (b) After 4 weeks, H\&E staining showed hPDL cells filled up the spaces in gelform and present in the center of the HA-PADM scaffold. Immnuohistochemical study with lower power showed cells lined up with the gelform framework. In the HA-PADM picture, cells were present on both sides of the HA-PADM scaffold, as well as in the center of the material. (c) Confocal microscopy imagining reviewed the hPDL cells grown inside of the scaffold, and spread along the direction of the collagen fibers. HA-PADM, hydroxyapatite-porcine acellular dermal matrix; H\&E, hematoxylin and eosin 
the surface compared with the cells on HA-PADM that had more processes and communicated with each other in three-dimensional. Since we also observed a higher F-actin expression level in cells on HA-PADM, we believe that the cell behavior with HA-PADM scaffold is closer to its nature. Further more, the mechanical properties of Gelfoam is not optimized for periodontal regeneration. With prolonged culture for 4 weeks, it is clearly observed that the PDL cells were able to migrate in to the center of the HA-PADM scaffold, and in some places, cells filled the channels in scaffolds.

Take biodegradation into consideration, the degradation test was done and the results illustrated that degradation rate of HAPADM is the slowest of all, that is to say, without significant weight change after 4 weeks' culture in vitro. In order to promote the periodontal regeneration, the weight of periodontal scaffolds should not change significantly in the first 6 weeks. This also demonstrated that the mechanical properties of HA-PADM are good enough to be used as scaffolds in vivo changed into in vitro. With the present of collagenase and other enzymes in the microenvironment at the peridontium, the condensed structure of HA-PADM could be a plus for tissue regeneration. With further modifications of HA-PADM scaffold, in order to provide more openings to allow cells grow in, we believe HA-PADM could potentially be used as a cell carrier for periodontal tissue regeneration. The next phase of the investigation will involve in vivo evaluation of the HA-PADM scaffold on the ability of delivery cells, maintaining space and timely degradation in periodontal defects.

\section{CONCLUSION}

The concepts of periodontal regeneration research demand tissue engineering with high cytocompatibility, guiding channel-like structure scaffolds. Our study showed that hPDL cells proliferated and migrated into the novel developed HA-PADM scaffold. The cell behavior was positively influenced by the scaffold. The properties of this acellularized, HA-treated porcine collagen scaffold make it a promising biomaterial to use as a cell carrier in periodontal regeneration.

\section{ACKNOWLEDGEMENTS}

This study is supported by Chinese post-doctoral fund (20090451410) and International cooperation program of science of Shandong Province (2011HZ035). We acknowledge the assistance of Dr Wade Sigurdson at the Confocal Microscope and Flow Cytometry Facility in the School of Medicine and Biomedical Sciences, and Dr Peter Bush at the Scanning Electron
Microscope Facility in the School of Dental Medicine, State University of New York at Buffalo.

1 Schroder NW, Meister D, Wolff $\mathrm{V}$ et al. Chronic periodontal disease is associated with single nucleotide polymorphisms of the human TLR-4 gene. Genes Immun 2005; 6(5): 448-451.

2 Deepa D, Mehta DS, Puri VK et al. Combined periodontic-orthodontic-endodontic interdisciplinary approach in the treatment of periodontally compromised tooth. J Indian Soc Periodontol 2010; 14(2): 139-143.

3 Karring T, Nyman S, Gottlow J et al. Development of the biological concept of guided tissue regeneration—animal and human studies. Periodontol 1993; 1(1): 26-35.

4 Cho EH, Park JC, Cha JK et al. Dimensional change of the healed periosteum on surgically created defects. J Periodontal Implant Sci 2011; 41(4): 176-184.

5 Giannopoulou C, Cimasoni G. Functional characteristics of gingival and periodontal ligament fibroblasts. J Dent Res 1996; 75(3): 895-902.

6 Wang LH, Greenwell H, Fiorellini J et al. Periodontal regeneration. J Periodontol2005; 76(9): 1601-1622.

7 Chen FM, Zhao YM, Zhang $\mathrm{R}$ et al. Periodontal regeneration using novel glycidyl methacrylated dextran (Dex-GMA)/gelatin scaffolds containing microspheres loaded with bone morphogenetic proteins. J Controlled Release 2007; 121(1/2): 81-90.

8 Yang $\mathrm{ZH}$, Jin $\mathrm{F}$, Zhang $\mathrm{XJ}$ et al. A novel possible strategy based on self-assembly approach to achieve complete periodontal regeneration. J Artificial Organs 2010; 34(7): 603-609.

9 Seo BM, Miura M, Gronthos S et al. Investigation of multipotent postnatal stem cells from human periodontal ligament. J Lancet 2004; 364(9429): 149-155.

10 Lin CC, Ritch R, Lin SM et al. A new fish scale-derived scaffold for corneal regeneration. J Eur Cells Mater 2010; 19: 50-57.

11 Lee CH, Singla A, Lee Y. Biomedical applications of collagen. Int J Pharm 2001; 221(1/2): 1-22.

$12 \mathrm{Ma} \mathrm{L}$, Gao C, Mao Z et al. Enhanced biological stability of collagen porous scaffolds by using amino acids as novel cross-linking bridges. Biomaterials 2004; 25(15): 2997 3004.

13 Hoerstrup SP, Sodian R, Daebritz S et al. Functional living trileaflet heart valves grown in vitro. Circulation 2000; 102 (19 Suppl 3): III44-III49.

14 Shinoka T, Shum-Tim D, Ma PX et al. Creation of viable pulmonary artery autografts through tissue engineering. J Thorac Cardiovasc Surg 1998; 115(3): 536-546.

15 Stock UA, Nagashima M, Khalil PN et al. Tissue-engineeredvalved conduits in the pulmonary irculation. J Thorac Cardiovasc Surg 2000; 119(4): 732-740.

16 Steinhoff $\mathrm{G}$, Stock $U$, Karim $\mathrm{N}$ et al. Tissue engineering of pulmonary heart valves on allogenic acellular matrix conduits: in vivo restoration of valve tissue. Circulation 2000; 102 (19 Suppl 3): III50-III55.

17 Joshi P, Chung C, Aukhil I et al. Endothelial cells adhere to the RGD domain and the fibrinogen-like terminal knob of tenascin. J Cell Sci 1993; 106 (Pt 1): 389-400.

18 Ngangan AV, McDevitt TC. Acellularization of embryoid bodies via physical disruption methods. Biomaterials 2009; 30(6): 1143-1149.

19 Niederkorn JY. Immune mechanisms of corneal allograft rejection. Curr Eye Res 2007; 32(12): 1005-1016.

This work is licensed under a Creative Commons Attribution-NonCommercial-NoDerivative Works 3.0 Unported License. To view a copy of this license, visit http:// creativecommons.org/licenses/by-nc-nd/3.0 